\title{
25 Research Soure \\ Habitat Suitability Study of Hylomecon Japonica Based on Maxent Model in China
}

\section{Cao Zhen}

School of Pharmacy, Xi'an Jiaotong University, Xi'an 710061, P. R. China https://orcid.org/0000-00015179-1672

\section{Zhang Xiaoyan}

Xian Jiaotong University: Xi'an Jiaotong University

\section{Xue Xuanji}

Xian Jiaotong University: Xi'an Jiaotong University

\section{Zhang Lei}

Xian Jiaotong University: Xi'an Jiaotong University

\section{Zhan Guanqun}

Xian Jiaotong University: Xi'an Jiaotong University

\section{Zhang Xinxin}

Xian Jiaotong University: Xi'an Jiaotong University

\section{Guo Zengjun ( $\nabla$ guozj@xjtu.edu.cn )}

School of Pharmacy, Xi'an Jiaotong University, Xi'an 710061, P. R. China

\section{Research}

Keywords: Hylomecon japonica, Regionalization of traditional Chinese medicine, Maxent, ArcGIS, Habitat suitability areas

Posted Date: September 21st, 2020

DOI: https://doi.org/10.21203/rs.3.rs-76168/v1

License: (c) (i) This work is licensed under a Creative Commons Attribution 4.0 International License.

Read Full License 


\section{Abstract}

Background: To understand the potential distribution and habitat suitability of $\mathrm{H}$. japonica in China. And to provide guidance for the wild cultivation and standardized planting of $H$. japonica.

Methods: The maximum entropy model (Maxent) and geographic information system (ArcGIS) were applied to predict the potential suitable habitat of $H$. japonica species, and the contribution of variables were evaluated by using the jackknife test.

Results: The AUC value confirmed the accuracy of the model prediction based on 101 occurrence records. The potential distributions of $H$. japonica were mainly concentrated in Jilin, Liaoning, Shaanxi and other provinces (adaptability index>0.6). Jackknife experiment showed that the precipitation of driest month (35.6\%), precipitation of wettest quarter (13.4\%), the mean annual temperature (7.8\%) and the subclass of soil $(7.8 \%)$ were the most important factors affecting the potential distribution of $\mathrm{H}$. japonica.

Conclusion: The niche parameters of the most suitable growth area (adaptability index>0.8) for $H$. japonica were precipitation of driest month $(5 \mathrm{~mm}$ ), precipitation of wettest quarter $(400-490 \mathrm{~mm})$, the mean annual temperature $\left(-2-4{ }^{\circ} \mathrm{C}\right)$ and the subclass of soil (Glossy Chernozem, Gleyic Lime, Haplic Gypsisols).

\section{Background}

Hylomecon japonica (Thunb.) Prantl et Kundig, a medicinal plant of the Papaveraceae family, is widely distributed in Shaanxi, Liaoning, Chongqing, Shanxi, Sichuan, Heilongjiang, and Henan in China, along with Japan and Korea[1]. It is a kind of perennial herb with irregular serrated leaves, and characterized by bright yellow flowers[2]. Meanwhile, its roots have medicinal potential, so it is commonly used in folk to treat diseases such as arthritis, neuralgia, and eczema[3]. Various alkaloids, phenols, flavonoids, saponins and other active compounds isolated from $\mathrm{H}$. japonica have been reported. And a new species of endophytic bacteria belonging to sphingomonas was isolated from the rhizome of $H$. japonica[4-6]. Although many pharmacological studies, including anti-inflammatory, anticancer and antibacterial activities, have been reported[7-9], the investigation of the medicinal value and mechanism of $H$. japonica is far from enough. Due to the important medicinal value of the species, the wild resources of $H$. japonica have been greatly destroyed, and in combination with the climate change, the natural habitat of these plants have been gradually reduced. And most of the previous studies focused on the chemical composition of $H$. japonica, while other studies focused on the mechanism of its pharmacological action. However, up to now, little information is available on the guidance to protect, develop and utilize the $H$. japonica resources.

The studies for regionalization of Traditional Chinese medicine (TCM), have been performed to analyze the relationship between the distribution of TCM resources and their surrounding environmental variables. Relevant factors such as ecological environment, geographical distribution, regional characteristics and quality of the TCM were classified. The research on regionalization of TCM began in 
1990s. And with the rapid development of science and technology, the research methods of TCM regionalization are gradually improved[10]. The Species Distribution Model (SDM) is the main model used to predict suitable habitats for species in the regionalization of TCM. At present, there are a large number of SDMs and related software that can draw suitable habitat distribution maps of species based on the observed distribution data. The potential distribution of Nelumbo nucifera Gaertn. was assessed in China by the GARP and Maxent[11]; Based on CLIMEX ecological software, the data were compiled and processed to predict the adaptive area of $V$. taliense Loes. F.[12]; And the BIOCLIM niche model have been used to predict the potential adaptability of Yulania Liliflora (Desr.) D. L, Fu[13]. Among these models, the Maxent model has shown many advantages over other models when applied to "existence-only" species occurrence data, and is widely used for species distribution prediction and habitat suitability assessment.

Combined with some other software such as ArcGIS, Maxent can extract factor variables according to the geographic information of sample collection points to analyze the habitat suitability of species. Furthermore, potentially suitable habitats can be divided into a specified number of levels as needed. Many reports have showed that the Maxent model has been used to analyze the habitat suitability of medicinal plants and ecologically important species in the study of regionalization of TCM. Based on Maxent model and GIS technology, the production regionalization of Angelica in China was studied[14]. By constructing Maxent model, Wang Dan predicted that the high adaptability areas of Bupleurum marginatum were mainly distributed in the border of provinces in the southern region[15]. The spatial analysis function of ArcGIS and Maxent model were used to predict the ecological and quality suitable areas of Gentianae Macrophyllae Radix in China[16]. And the Maxent and ArcGIS were also used to predict the production regionalization and its suitability level of Paris polyphylla Smith var. chinensis (Franch.) Hara. in China[17]. The Maxent model only needs a set of known data (such as longitude and latitude information of sampling points) and variable factors, such as topography, precipitation, soil, temperature, vegetation type. These continuous or classified data are used to predict the habitat suitability distribution by combining the interactions between variables. The model has the characteristics of good performance with incomplete datasets, high efficiency, easy operation, high accuracy, small sample size requirements and high guiding significance[18].

To seek suitable habitats and evaluate the great practical significance for the development of $H$. japonica production, rational development and utilization and protection of $H$. japonica resources. In the present study, the occurrence records of $H$. japonica were collected for habitat suitability assessment. We used Maxent to simulate the suitability of $H$. japonica habitats on the occurrence records, based on variable factors (including soil, topography, precipitation, bioclimatic variables, temperature, and vegetation type), and analyzed the potential suitable habitats of $H$. japonica by means of ArcGIS. Maxent model evaluates the accuracy of model calculation results according to receiver-operating characteristic (ROC) curve (AUC), and the key environmental variables related to the geographical distribution of $H$. japonica were mapped by Jackknife test.

\section{Materials And Methods}




\subsection{Species data}

In this study, A total of 101 occurrences of $H$. japonica were collected from the following sources: (1) the Global Biodiversity Information Facility Data Portal (GBIF, https://www.gbif.org/); (2) the Specimen Resources Sharing Platform for Education (http://mnh.scu.edu.cn/); (3) the Chinese Virtual Herbarium (CVH: http://www.cvh.org.cn/). (4) Chinese plant species information System (http://www.iplant.cn/); (5) relevant literature and field investigation. In addition, only one was retained when the obtained geographic information of the sample repeated. When the obtained records lacked the required geographic information, Google Earth 7.0 would be used to obtain approximate latitude and longitude information based on the described geographic location. The geographic information of each sampling point would be converted into geographic coordinates (World Geodetic System 1984 data) through ArcGIS (version 10.2) (http://www.esri.com/). Finally, there were a total of 101 valid points, and the sample points were mainly from Liaoning, Shaanxi, Chongqing, Shanxi, Sichuan, and Heilongjiang in China. According to the requirements of Maxent software, the distribution records of the $H$. japonica species were converted into ".csv" format files. The vector map of China's administrative divisions with province boundaries provided by the National Basic Geographic Information System (http://bzdt.ch.mnr.gov.cn/index.jsp) was used as the base map for analysis, and the specie's suitability distribution map was generated by ArcMap 10.2 (Fig. 1).

\subsection{Variables}

Through relevant literature[19-21], 54 ecological factor data, including meteorological factors, soil type and topographic data, which affect the distribution of Chinese medicinal materials were selected. Among them, meteorological factors played a key role in influencing the target plants' distribution, including 19 bioclimatic variables, precipitation variables and temperature variables, with a spatial resolution of $30 \mathrm{~s}$, were downloaded from the WorldClim-Global Climate Database (http://worldclim.org/). Additionally, soil factors have also been shown to be important factors affecting species distribution[22]. Thus, the soil variables which were obtained from Harmonized World Soil Database (https://iiasa.ac.at/) were used to establish the model. Finally, the Digital Terrain Model (DTM) with $1 \mathrm{~km}$ resolution, downloaded from the Data center of resources and environment science of Chinese Academy of Sciences (http://www.resdc.cn/), was used to generate the slope, aspect and elevation data layers (Table 1). In the projection coordinate system as "Asia_Lambert_Conformal_Conic", these variables were extracted into China and converted to ASCII files according to the unified processing scope and pixel size. 
Table 1

variables used in the study in predicting the distribution of $H$. japonica

\begin{tabular}{|c|c|c|c|}
\hline Classification & Factors & Name & Unit \\
\hline \multirow{11}{*}{$\begin{array}{l}\text { The temperature } \\
\text { factors }\end{array}$} & bio1 & Mean annual temperature & ${ }^{\circ} \mathrm{C}$ \\
\hline & bio2 & Mean of monthly & ${ }^{\circ} \mathrm{C}$ \\
\hline & bio3 & Isothermality (bio2 / bio7 × 100) & 1 \\
\hline & bio4 & SD of temperature seasonality & 1 \\
\hline & bio5 & Max temperature of warmest month & ${ }^{\circ} \mathrm{C}$ \\
\hline & bio6 & Min temperature of coldest month & ${ }^{\circ} \mathrm{C}$ \\
\hline & bio7 & Temperature annual range (bio5-bio6) & ${ }^{\circ} \mathrm{C}$ \\
\hline & bio8 & Mean temperature of wettest quarter & ${ }^{\circ} \mathrm{C}$ \\
\hline & bio9 & Mean temperature of driest quarter & ${ }^{\circ} \mathrm{C}$ \\
\hline & bio10 & Mean temperature of warmest quarter & ${ }^{\circ} \mathrm{C}$ \\
\hline & bio11 & Mean temperature of coldest quarter & ${ }^{\circ} \mathrm{C}$ \\
\hline \multirow{8}{*}{$\begin{array}{l}\text { Precipitation } \\
\text { factors }\end{array}$} & bio12 & Annual precipitation & $\mathrm{mm}$ \\
\hline & bio13 & Precipitation of wettest month & $\mathrm{mm}$ \\
\hline & bio14 & Precipitation of driest month & $\mathrm{mm}$ \\
\hline & bio15 & $\begin{array}{l}\text { Coefficient of variation of precipitation } \\
\text { seasonality }\end{array}$ & 1 \\
\hline & bio16 & Precipitation of wettest quarter & $\mathrm{mm}$ \\
\hline & bio17 & Precipitation of driest quarter & $\mathrm{mm}$ \\
\hline & bio18 & Precipitation of warmest quarter & $\mathrm{mm}$ \\
\hline & bio19 & Precipitation of coldest quarter & $\mathrm{mm}$ \\
\hline \multirow{2}{*}{$\begin{array}{l}\text { Meteorological } \\
\text { factors }\end{array}$} & pre1-pre12 & Monthly precipitation from January to December & $\mathrm{mm}$ \\
\hline & $\begin{array}{l}\text { tmean1- } \\
\text { tmean12 }\end{array}$ & $\begin{array}{l}\text { Average monthly temperature from January to } \\
\text { December }\end{array}$ & ${ }^{\circ} \mathrm{C}$ \\
\hline \multirow[t]{3}{*}{ Soil factors } & ph & T_PH_H $\mathrm{H}_{2} \mathrm{O}$ (The soil reaction of topsoil) & 1 \\
\hline & cec & $\begin{array}{l}\text { T_CEC_SOIL (The cation exchange capacity in } \\
\text { topsoil) }\end{array}$ & $\mathrm{cmol} / \mathrm{kg}$ \\
\hline & sand & T_SAND (Percentage sand in the topsoil) & $\%$ \\
\hline
\end{tabular}

/*Is represented as a category variable. 


\begin{tabular}{|c|c|c|c|}
\hline Classification & Factors & Name & Unit \\
\hline & clay & $\begin{array}{l}\text { T_CLAY (Percentage clay respectively in the } \\
\text { topsoil) }\end{array}$ & $\%$ \\
\hline & sym90 & $\begin{array}{l}\text { SU_SYM90 (Name of soil in FAO90 Soil } \\
\text { Classification System) }\end{array}$ & $l^{*}$ \\
\hline & awc & AWC_CLASS (Soil available water content grade) & $I^{*}$ \\
\hline & usda & T_USDA_TEX (Texture class name and code) & $1^{*}$ \\
\hline & oc & $\begin{array}{l}\text { T_OC (The percentage of organic carbon in } \\
\text { topsoil) }\end{array}$ & $\%$ \\
\hline \multirow[t]{3}{*}{ Terrain factors } & dem & Alt & $\mathrm{m}$ \\
\hline & slope & Slope & 。 \\
\hline & aspect & Aspect & $\rho^{*}$ \\
\hline
\end{tabular}

\subsection{Modeling procedure}

Based on the geographic data and 54 variable factors obtained, Maxent software (Version 3.4.1: http://www.cs.princeton.edu/schapire/maxent) was used to calculate the habitat suitability of $H$. japonica. Parameter setting of modeling was as follows: Among the occurrence records of $H$. japonica, $20 \%$ were randomly selected as test data and the remaining $80 \%$ were used as training data. Response curves were created when all the variables with ".asc" format were selected as environmental layers. And a jackknife test was performed to measure the importance of each variable to the model, and the ROC curve was used to test the accuracy of the prediction results. Finally, all other settings were kept as default value[23].

\section{Results}

\subsection{Habitat suitability distribution}

Figure 1 showed the habitat suitability distributions of $\mathrm{H}$. japonica in China, according to the combination technology of Maxent and ArcGIS software. The habitat suitability results were expressed as probability with a range of [0-1][24]. Using the reclassification tool of ArcMap 10.2, the probability results obtained were divided into four levels, of which 0-0.2 was considered unsuitable, 0.2-0.4 was considered generally suitable, $0.4-0.6$ was considered moderately suitable, and 0.6-1 was considered as highly suitable[25].

The analysis results showed that the regions highly suitable for $H$. japonica were mainly distributed in the southeast of Heilongjiang, Jilin, Liaoning and Shandong Provinces, as well as the east of Shaanxi, Shanxi, Gansu, Ningxia. And the junction of Shaanxi with Henan, Sichuan, Hubei, and Chongqing, with 
relatively small distributions in Guizhou, Hunan, Jiangxi and Fujian. The moderately suitable areas for $H$. japonica were found to be mainly distributed around the highly suitable areas of $H$. japonica. And the generally suitable areas for $\mathrm{H}$. japonica were found to be distributed in Guizhou, Sichuan, and Heilongjiang Province, with sporadic distributions in Anhui, Jiangsu, Zhejiang, Henan, and a small part of Xinjiang and Xizang (Fig. 1).

\subsection{Accuracy test}

The area under receiver-operating characteristic (ROC) curve (AUC) obtained by the accuracy test of the ROC curve analysis method was used in the Maxent model performance evaluation. The AUC values were between 0 and 1 , and divided into six parts. When the AUC value was lower than 0.5 , the model executed was worse than contingency. When the AUC value ranges from 0.5 to 0.6 , the model performance was poor, 0.6-0.7 was fair, 0.7-0.8 was good, 0.8-0.9 was very good, and 0.9-1 was excellent[26]. Figure 2 showed that the AUC value predicted by Maxent based on the variables of potential $H$. japonica distribution was 0.938 , which indicated that Maxent model has an excellent prediction effect on the potential distribution region of $H$. japonica in China.

\subsection{Contribution of environmental variables}

The relative contributions of variables to the Maxent model under current circumstances obtained by Maxent iterative calculation and the results of jackknife analyses obtained the main variable factors influencing the $H$. japonica distribution and their contribution rates. Variables affecting the $H$. japonica distribution can be obtained as follows: precipitation of driest month (BI014), precipitation of wettest quarter (BIO16), mean annual temperature (BIO1), subclass of soil (SU_SYM90), altitude (DEM) and mean temperature of driest quarter (BIO9). The total contribution of these variables was $76.2 \%$, indicating that the $H$. japonica distribution was strongly influenced by these variables. The total contribution rate of the 19 bioclimatic variables was $74.9 \%$. The total contribution rate of the 8 soil variables was $12.7 \%$. The contribution rate of the three terrain variables was $11.5 \%$. This indicates that the influence of bioclimatic variables on $\mathrm{H}$. japonica habitat distribution is much greater than that of topography and soil.

The relationship between the precipitation in the driest month (Fig. 4A) and the probability of the existence of $H$. japonica indicated that when the precipitation in the driest month was about $5 \mathrm{~mm}$, the probability of the existence of $H$. japonica could reach $80 \%$. When precipitation in the driest months was between $4 \mathrm{~mm}$ and $35 \mathrm{~mm}$, the probability of $H$. japonica was higher than $50 \%$. The precipitation of wettest quarter also exerted an enormous function on the distribution of $H$. japonica (Fig. 4B). When the precipitation of wettest quarter ranged between $375 \mathrm{~mm}$ and $640 \mathrm{~mm}$, the presence probability of $H$. japonica occurrence was greater than $50 \%$, when the precipitation of wettest quarter was $400-490 \mathrm{~mm}$, the presence probability of $H$. japonica could reach $80 \%$ or more. And then, mean annual temperature was also an important bioclimatic variable which effect the distribution of $H$. japonica (Fig. 4C). When the mean annual temperature ranged from -7.5 to $7.5^{\circ} \mathrm{C}$, the presence probability of $\mathrm{H}$. japonica occurrence was greater than $50 \%$, when the mean annual temperature was in the range of $-2-4{ }^{\circ} \mathrm{C}$, the presence 
probability of $H$. japonica could reach $80 \%$ or more. Finally, mean temperature of driest quarter was another bioclimatic variable that affected the distribution of $H$. japonica (Fig. 4D). When the mean temperature of driest quarter ranged from 13 to 23.7 , more than $50 \%$ probability of $H$. japonica could be achieved, when the mean temperature of driest quarter was 21.2 , the presence probability of $H$. japonica could reach $80 \%$.

Among all of the soil variables, subclass of soil played a vital role in the distribution of $H$. japonica. In the ACRISOLS, Glossic Chernozems, Luvic Calcisols, Petric Calcisols, Gleyic Greyzems and Haplic Gypsisols (Fig. 4E) soils the presence probability of $H$. japonica were more than $50 \%$.

Altitude also played an important role in influencing the distribution of $H$. japonica, with its maximum value being $500 \mathrm{~m}$ (Fig. 4F), at which time the probability of $H$. japonica existence could reach more than $80 \%$. As the height decreased or increased from $500 \mathrm{~m}$, the occurrence probability of $H$. japonica decreased gradually.

\section{Discussion}

Traditional Chinese medicine, as a traditional medicinal plant in China, plays a pivotal role in the country's medical and health services. The scientific research of Chinese medicinal materials is different from the research of general chemical and biological medicine. Its survival rate and quality are the result of multiple variables, which are related to many ecological factors (such as temperature, rainfall, soil, topography). Therefore, blind cultivation and planting when the suitability of the production area is not clear may cause problems such as a decrease in the survival rate of the medicinal materials and a decrease in the main medicinal components. In recent years, the study for regionalization of TCM has become more and more important in the study of the distribution and cultivation of Chinese medicine. The Maxent model combined with ArcGIS has developed into an important tool for evaluating the influence of various variables on species distribution in recent years. They are based on species existence records and various variable factors that may affect the species distribution, and analyze their correlations to predict the current potential distribution of species. The regionalization studies of various medicinal plants such as Artemisia annua L.[18], Coptis chinensis[10], Bupleurum marginatum[15] and Rhodiola kirilowi[27] have been carried out successively, and have shown high accuracy. H. japonica is a valuable medicinal plant with a wide range of pharmacological effects such as antibacterial, antiinflammatory, anti-cancer and other pharmacological functions, and its material basis is mainly a variety of alkaloids. This is the first time to analyze and simulate the distribution of $H$. japonica based on the Maxent model, which can provide a basis for the protection and cultivation of $H$. japonica.

In present study, 54 variables combined with 101 existence records were systematically used to establish the Maxent models of $H$. japonica species. The results of the prediction model showed that the habitat suitability of $H$. japonica agreed strongly with the data set of $H$. japonica occurrence. And the Jackknife test of variables revealed that precipitation of driest month, precipitation of wettest quarter, mean annual temperature, subclass of soil, altitude and mean temperature of driest quarter, had high contributions to 
the distribution of $H$. japonica. The precipitation of driest month showed the highest contribution (35.6\%), followed by the precipitation of wettest quarter with the contribution of $13.4 \%$. The mean annual temperature is another important variable that can explain the distribution of $H$. japonica, and the subclass of soil provides almost the same contribution (7.8\%). These results are consistent with the habitat characteristics of the existing distribution of $H$. japonica. From the results of the study, the characteristics of the ecological environment factors in the best area of $H$. japonica distribution are: the precipitation of driest month $(5 \mathrm{~mm})$, the precipitation of wettest quarter $(400-490 \mathrm{~mm})$, the mean annual temperature $\left(-2-4{ }^{\circ} \mathrm{C}\right.$ ) and the subclass of soil (Glossic Chernozems, Gleyic Greyzems, Haplic Gypsisols). The results indicated that $H$. japonica species could be introduced into many undiscovered potential regions, such as Shandong and Guizhou.

This study can provide scientific basis for the cultivation and planting of $H$. japonica in China. However, before large-scale planting, field trials must be carried out because the yield and quality of medicinal plants can be affected by many other factors, such as existing species in the introduced area, natural disasters and road traffic. According to our results, this method could be used to predict the potential distribution of other medicinal plants and provide a valuable tool for species conservation and distribution research. However, there are still some aspects to be improved in this study. First of all, the potential distribution area predicted by Maxent model always overestimates the actual niche of the species, because the species may be unable to disperse to the possible areas due to human interference, topographical obstacles, and species competition. Secondly, in addition to the selected variables in this study, other variables may also affect the distribution range of $H$. japonica. Finally, the combination of multi-effect components with quality evaluation will make the study of $H$. japonica regionalization more comprehensive and reasonable.

\section{Conclusion}

In this study, a large number of occurrence data sets for $H$. japonica species were obtained from China, and a potential distribution model of $H$. japonica species based on multiple variables was successfully established. The habitat suitability of $H$. japonica was successfully simulated and the main variables affecting the distribution were determined. The results indicated that the precipitation of driest month, the precipitation of wettest quarter, the mean annual temperature and the subclass of soil were important factors which could control the distribution of $H$. japonica species. This provides a reference for the protection, introduction and cultivation of $H$. japonica in ecologically suitable areas. However, in addition to the variables that have been included, other factors (including biological factors, vegetation types, and human activities) may also affect the survival rate and the main medicinal ingredient of medicinal plants, and may be key factors in the formation of metabolites of medicinal plants. Further studies involving more factors into the model will enhance the accuracy of the prediction of $H$. japonica distribution in China.

\section{Abbreviations}


TCM

Traditional Chinese medicine

SDM

Species Distribution Model

ROC

Receiver-operating characteristic

AUC

Area Under Curve

\section{Declarations}

\section{Authors' contributions}

CZ and GZJ conceived and designed the study and wrote the manuscript. ZXY provided research methods. All authors are responsible for reviewing data. All authors read and approved the final manuscript.

\section{Acknowledgements}

The authors are grateful for the financial support received from the fourth national survey on Chinese materia medica resources funded by subsidies for public health services of traditional Chinese medicine in 2018 (Caishe No. 2018 43). We thank the School of Pharmacy of Xi'an Jiaotong University and Shaanxi Traditional Chinese Medicine Extracts Engineering Technology Research Center for providing the necessary laboratory facilities.

\section{Competing interests}

The authors declare that they have no conflict of interest.

\section{Availability of data and materials}

The readers can use data and materials in this manuscript by quotation of author names and Journal of Chinese Medicine.

\section{Consent for publication}

All of authors consent to publication of this study in Journal of Chinese Medicine.

\section{Ethics approval and consent to participate}

Not applicable.

\section{Funding}


Project supported by the fourth national survey on Chinese materia medica resources funded by subsidies for public health services of traditional Chinese medicine in 2018 (Caishe No. 2018 43).

\section{References}

1. Xu X, Wang D. Are There Two Varieties in Hylomecon japonica (Papaveraceae)? Morphological and Molecular evidence. ANN BOT FENN. 2017;54(4-6):391-9.

2. Zhang Y, Lee J, Liu X, Sun Z. The first complete chloroplast genome of Hylomecon japonica and its phylogenetic position within Papaveraceae. Mitochondrial DNA Part B. 2019;4(2):2349-50.

3. Kim SW, In DS, Kim TJ, Liu JR. High frequency somatic embryogenesis and plant regeneration in petiole and leaf explant cultures and petiole-derived embryogenic cell suspension cultures of Hylomecon vernalis. Plant Cell Tissue Organ Cult. 2003;74(2):163-7.

4. Akbar A: Endophytic Sphingomonas hylomeconis sp. nov., isolated from the stem of Hylomecon japonicaa.: School of life science, Northwest Agriculture and Forestry University; 2016.

5. Lee SY, Kim KH, Lee IK, Lee KH, Choi SU, Lee KR. A new flavonol glycoside from Hylomecon vernalis. ARCH PHARM RES. 2012;35(3):415-21.

6. Qu Y, Gao J, Wang J, Geng Y, Zhou Y, Sun C, Li F, Feng L, Yu M, Wang G. New Triterpenoid Saponins from the Herb Hylomecon japonica. MOLECULES. 2017;22(10):1731.

7. Chae HS, Kang OH, Keum JH, Kim SB, Mun SH, Seo YS, Kim MR, Choi JG, Shin DW, Oh YC, et al. Antiinflammatory effects of Hylomecon hylomeconoides in RAW 264.7 cells. Eur Rev Med Pharmacol Sci. 2012;16(Suppl 3):121-5.

8. Lee H, Cho H, Yu R, Lee K, Chun H, Park J. Mechanisms Underlying Apoptosis-Inducing Effects of Kaempferol in HT-29 Human Colon Cancer Cells. INT J MOL SCI. 2014;15(2):2722-37.

9. Choi J, Kang O, Chae H, Obiang-Obounou B, Lee Y, Oh Y, Kim M, Shin D, Kim J, Kim Y, et al. Antibacterial Activity of Hylomecon hylomeconoides Against Methicillin-Resistant Staphylococcus aureus. APPL BIOCHEM BIOTECH. 2010;160(8):2467-74.

10. Liu X: Cultural regionalization for Coptis chinensis based on $3 S$ technology platform.: Hubei University of Chinese Medicine; 2015.

11. Lu-Dan C, Wan H, Dan-Qi L, Dong-Mei C. Prediction of suitable distribution areas of the endangered plant wild Nelumbo nucifera Gaertn. in China. Plant Science Journal. 2019;37(06):731-40.

12. Yin Z, Zhang J, Xie H: Study on the prediction of the Chinese medicinal plant $\mathrm{V}$. taliense Loes. $\mathrm{F}$ in

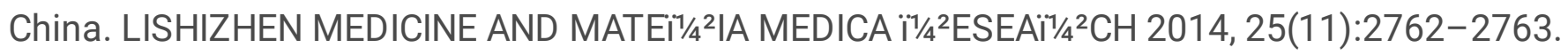

13. Li-Yong S, Zheng J, Cheng-Ni L, Zeng-Fang Y. Analysis of the adaptive and geographical distribution of Yulania liliflora based on DIVA-GIS. 2018.

14. Hui Y, Xiao-bo Z, Shou-dong Z, Da-wei Q, Lan-ping G, Lu-qi H. Jin-ao D: Production regionalization study of Chinese angelica based on MaxEnt model. China Journal of Traditional Chinese Medicine. 2016;41(17):3139-47. 
15. Wang D: Prediction of Bupleurum marginatum habitat suitability and influence of climate change on its spatial pattern.: SHAANXI NORMAL UNIVERSITY; 2017.

16. You-yuan LU, Xiao-bo Z, Yan-mei Y, Xiao-hui MA, Tian-tian Z, Xiao-hui YU, Ling J. Quality regionalization study on Gentianae Macrophyllae Radix. China Journal of Chinese Materia Medica. 2016;41(17):3132-8.

17. Chen $T$, Zhang $T$, Fang Q, Wen F, Yang $Y$, Zhang H, Xue D. Prediction of Paris polyphylla Smith var. chinensis (Franch.) Hara. habitat suitability based on MaxEnt and ArcGIS. Journal of Chinese Medicinal Materials. 2017;40(04):803-6.

18. Zhang Q: Quality variation and production regionalization of Artemisia annua L.: Chinese Academy of Medical Sciences, Peking Union Medical College; 2018.

19. Yang X, Kushwaha SPS, Saran S, Xu J, Roy PS. Maxent modeling for predicting the potential distribution of medicinal plant, Justicia adhatoda L. in Lesser Himalayan foothills. ECOL ENG. 2013;51:83-7.

20. Wang Y, Zhang L, Du Z, Pei J, Huang L. Chemical Diversity and Prediction of Potential Cultivation Areas of Cistanche Herbs. SCI REP-UK 2019, 9(1).

21. Sun H: Quantitative methodology on the quality assessment and functional regionalization evaluation of Notopterygii Rhizoma et Radix.: Guangdong Pharmaceutical University; 2016.

22. Song C, Liu H. Habitat differentiation and conservation gap of Magnolia biondii, M. denudata, and M.sprengeri in China. PEERJ. 2019;6:e6126.

23. Shen L, Xu J, Luo L, Hu H, Meng X, Li X, Chen S. Predicting the potential global distribution of diosgenin-contained Dioscorea species. CHIN MED-UK 2018, 13(1).

24. Remya K, Ramachandran A, Jayakumar S. Predicting the current and future suitable habitat distribution of Myristica dactyloides Gaertn. using MaxEnt model in the Eastern Ghats, India. ECOL ENG. 2015;82:184-8.

25. Li J, Fan G, He Y. Predicting the current and future distribution of three Coptis herbs in China under climate change conditions, using the MaxEnt model and chemical analysis. SCI TOTAL ENVIRON. 2020;698:134141.

26. Jiang H, Liu T, Li L, Zhao Y, Pei L, Zhao J. Predicting the Potential Distribution of Polygala tenuifolia Willd. under Climate Change in China. PLOS ONE. 2016;11(9):e163718.

27. Hong D: Study on the resources and quality analysis of Tibetan Medicine Rhodiola kirilowii.: Chengdu University of Traditional Chinese Medicine; 2018.

\section{Figures}




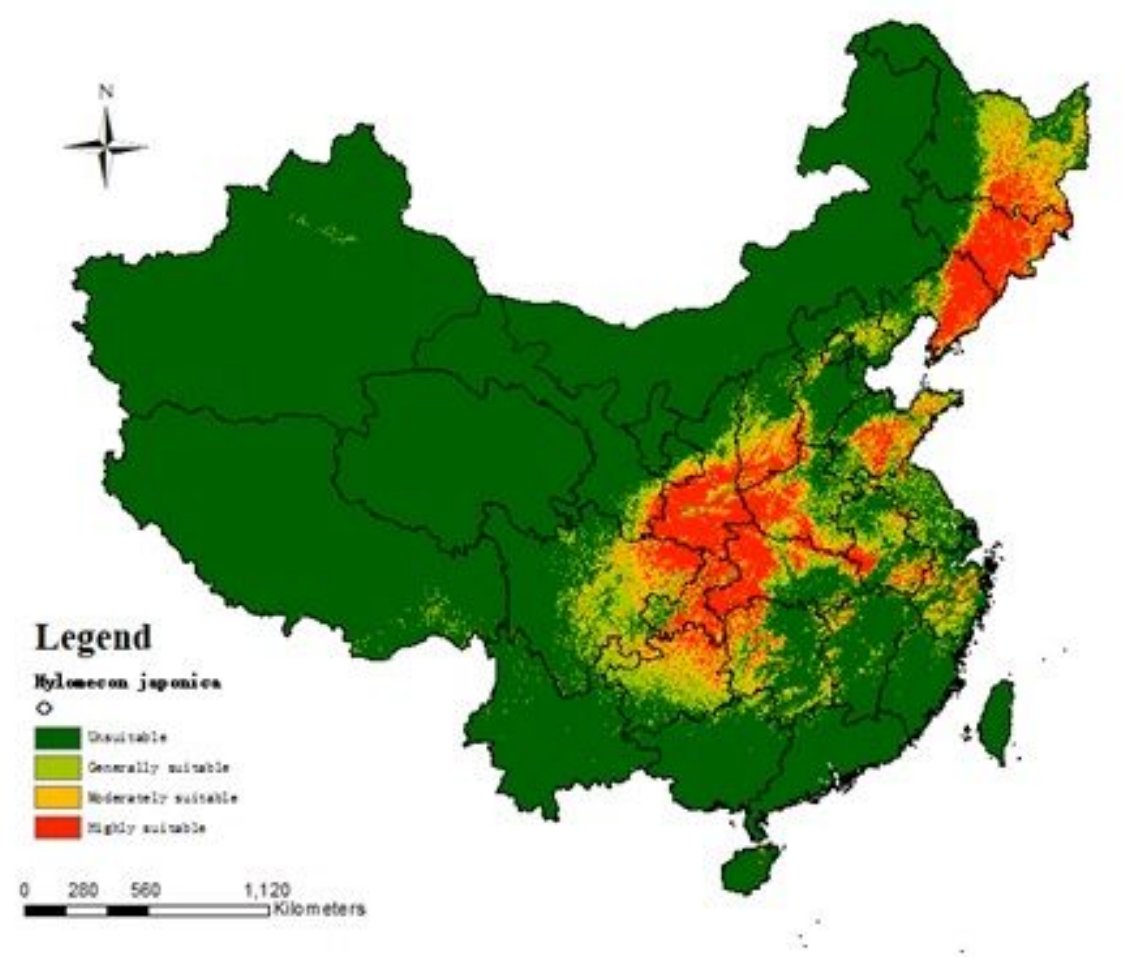

Figure 1

Predicted spatial distribution of $\mathrm{H}$. japonica in China based on Maxent. Note: The designations employed and the presentation of the material on this map do not imply the expression of any opinion whatsoever on the part of Research Square concerning the legal status of any country, territory, city or area or of its authorities, or concerning the delimitation of its frontiers or boundaries. This map has been provided by the authors.

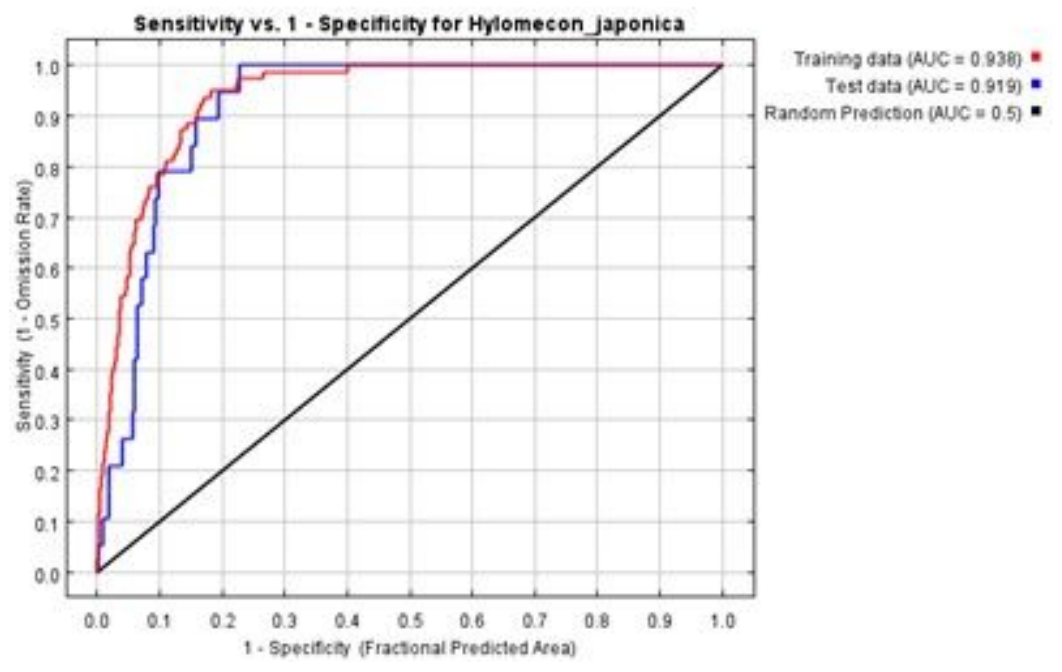

Figure 2

ROC curves of Maxent models for $\mathrm{H}$. japonica species. 

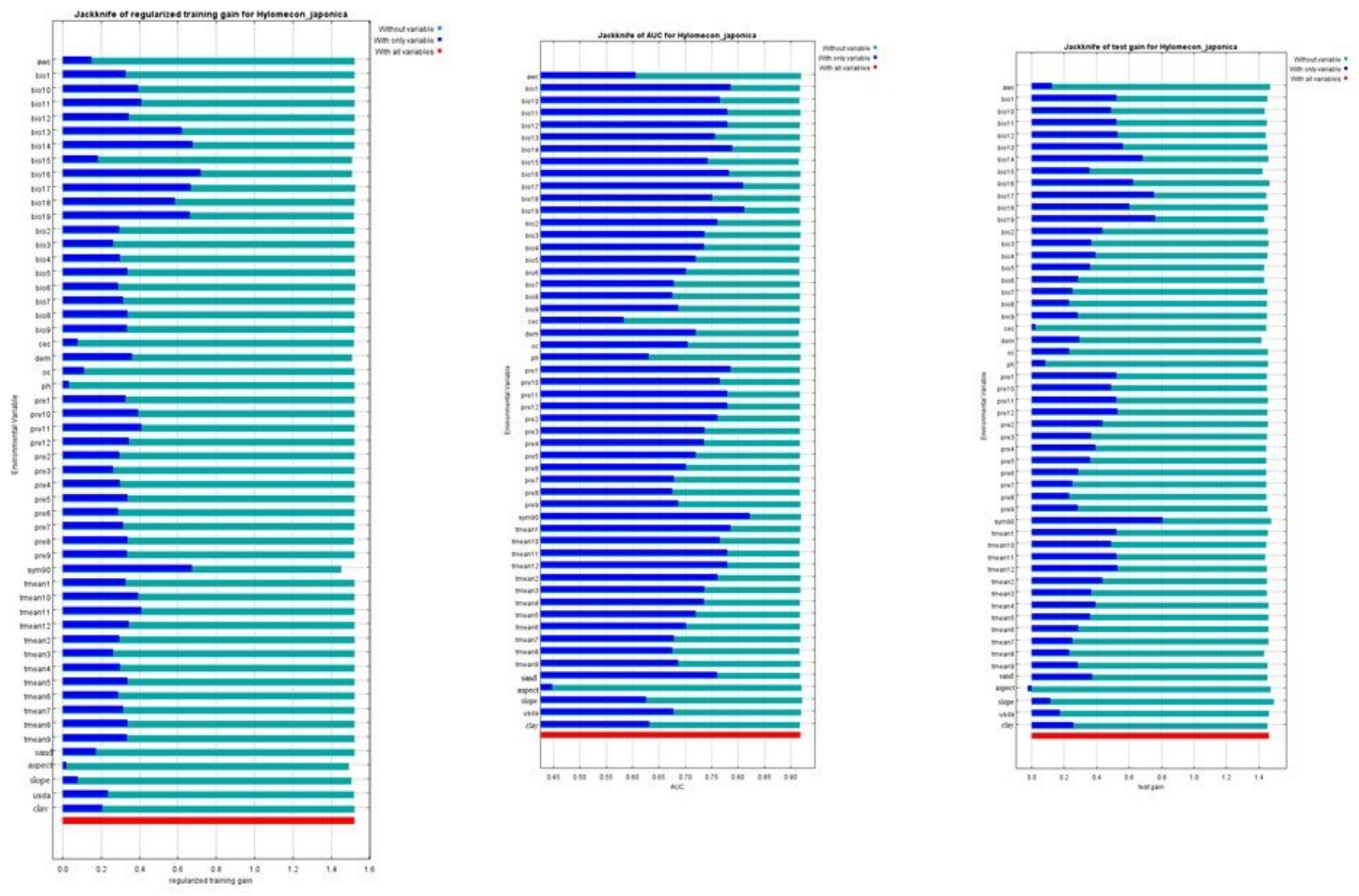

\section{Figure 3}

Results of the Jackknife Test of Variables' Contribution in Modeling H. japonica 's Potential Habitat Distribution. The graph shows the result of the Jackknife test of variable importance using regularized training gain, AUC and test gain on test data respectively. The blue bars indicate the gain using solo environmental variable, the green bars indicate the gain excluding the single variable from the full model, and the red bars indicate the gain considering all variables. 


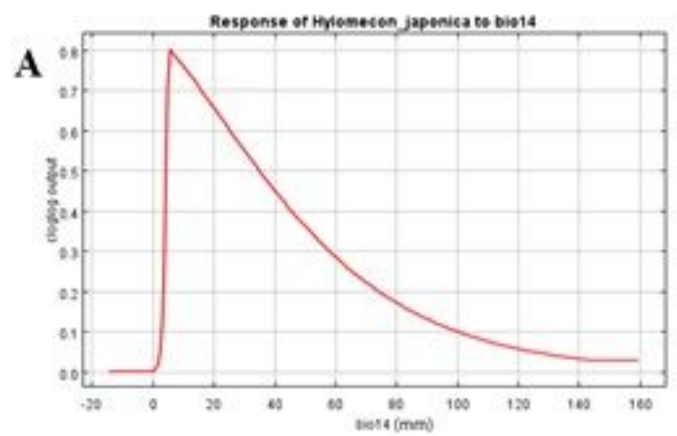

B
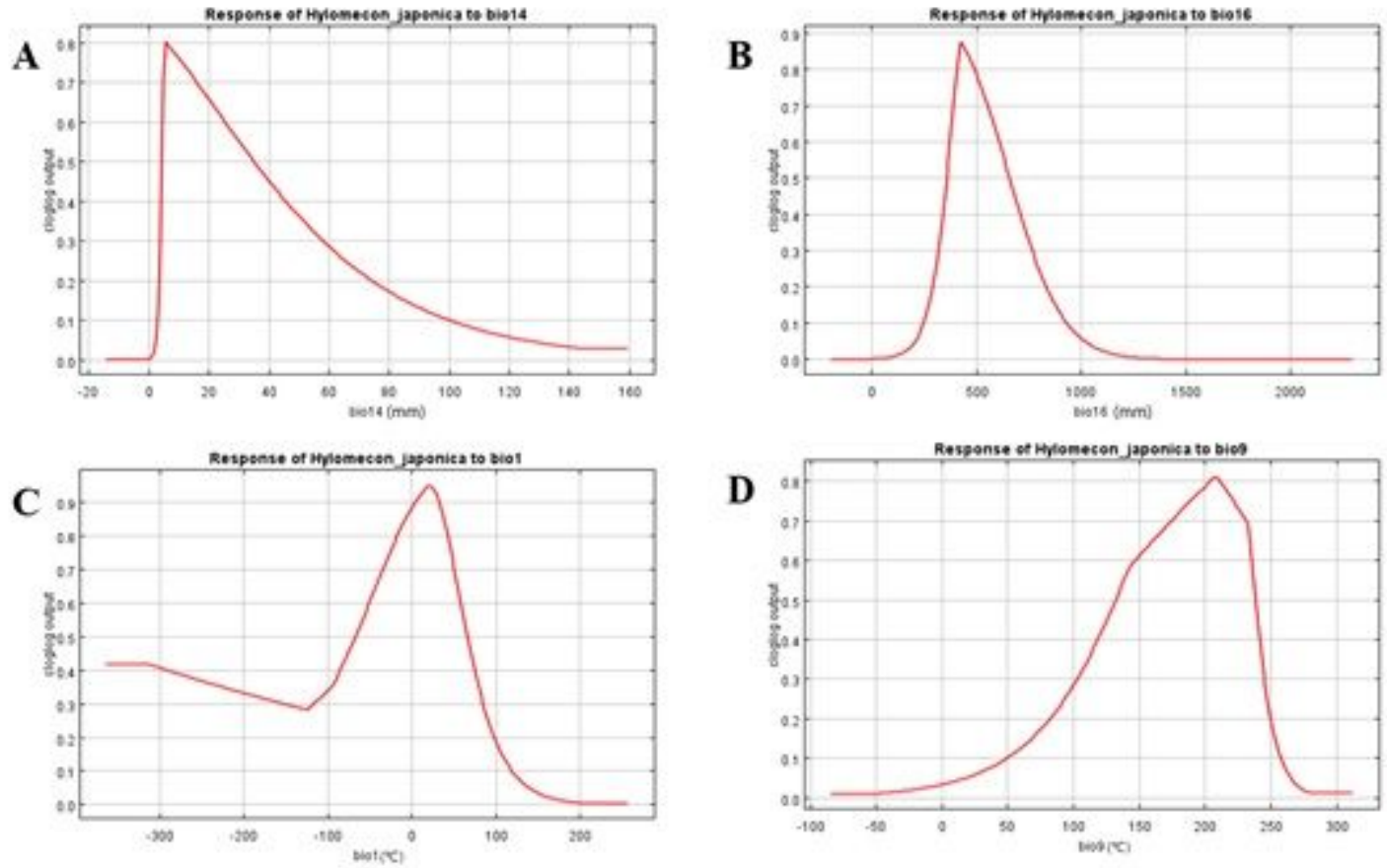

D
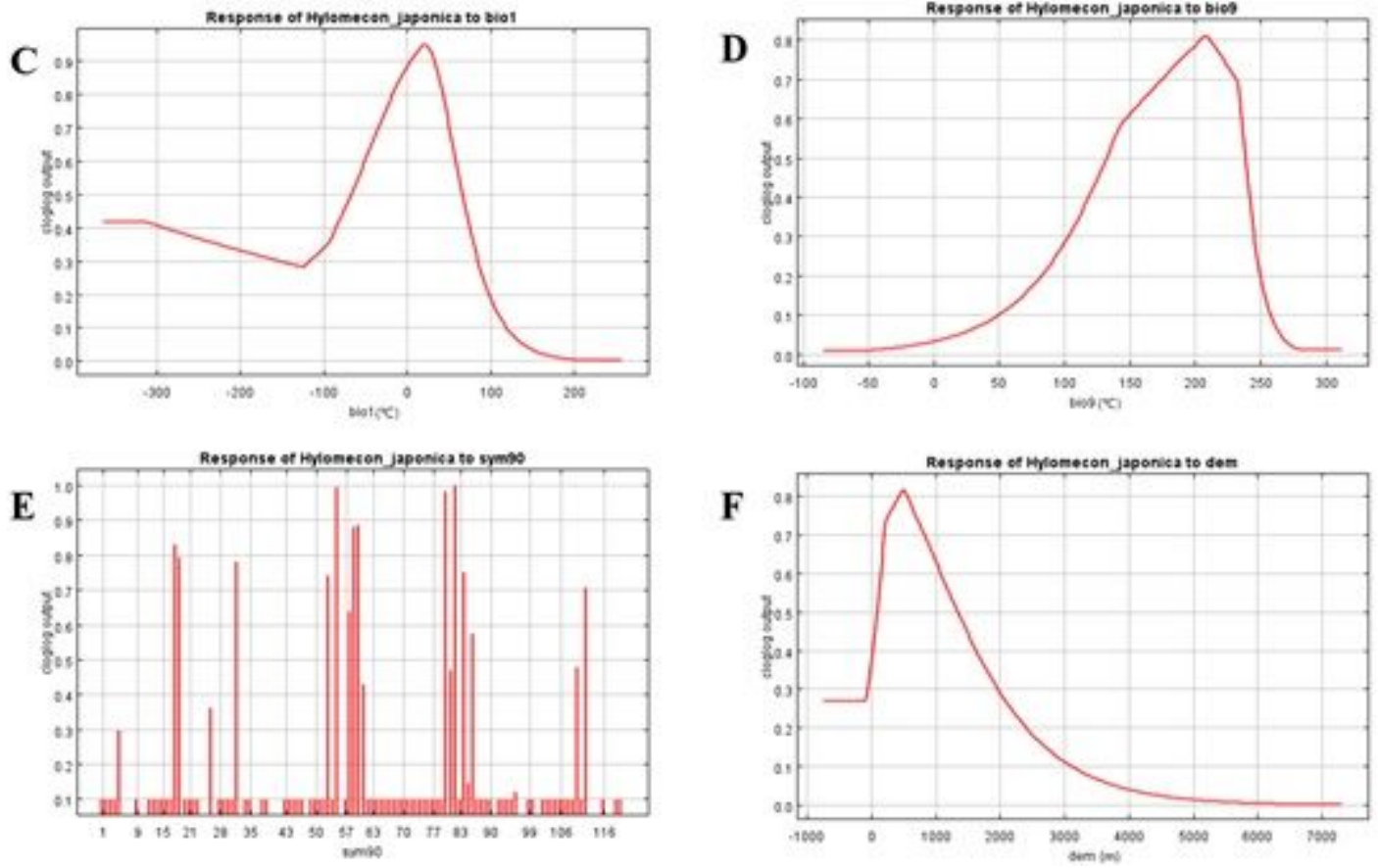

Figure 4

The response curves for the six largest contributing variables for $\mathrm{H}$. japonica. (A) Precipitation in the driest month, (B) Precipitation of wettest quarter, (C) Mean annual temperature, (D) Mean temperature of driest quarter, (E) Subclass of soil and (F) Altitude. 